\title{
Influence of nanoparticles of various types as fillers on resistance to hydrolysis of films of heat-resistant polyimide
}

\author{
E. N. Bykova ${ }^{1}$, I. V. Gofman ${ }^{1}$, E. M. Ivankova ${ }^{1}$, A. L. Nikolaeva ${ }^{1}$, \\ A. V. Yakimansky ${ }^{1,2}$, O. S. Ivanova ${ }^{3}$, A. E. Baranchikov ${ }^{3}$, V. K. Ivanov ${ }^{3,4}$ \\ ${ }^{1}$ Institute of Macromolecular Compounds, Russian Academy of Sciences, \\ 199004, Bolshoi prospect 31, Saint Petersburg, Russia \\ ${ }^{2}$ Saint Petersburg State University, Institute of Chemistry, \\ 198504, Universitetskii prospect 26, Peterhof, Saint Petersburg, Russia \\ ${ }^{3}$ Kurnakov Institute of General and Inorganic Chemistry, Russian Academy of Sciences, \\ 119991, Leninsky prospect 31, Moscow, Russia \\ ${ }^{4}$ Lomonosov Moscow State University, Faculty of Materials Science, \\ 119991, Leninskie gory 1, building 73, Moscow, Russia
}

bykova.elena.n@gmail.com,gofman@imc.macro.ru,ivelen@mail.ru, alexandra.l.nikolaeva@gmail.com, yakimansky@yahoo.com, runetta05@mail.ru, a.baranchikov@yandex.ru,van@igic.ras.ru

DOI 10.17586/2220-8054-2019-10-6-666-673

\begin{abstract}
Impact of nanoparticles of various types as fillers on the stability of the poly(pyromellitimide)-based nanocomposite films' properties in alkaline hydrolysis was studied. It was shown that the introduction of nanoparticles into the polymer can lead to an increase in excess free volume. This fact is evidenced by scanning electron microscopy and densitometric studies. The increase of the excess free volume was shown to provoke a rise of the diffusion intensity of the hydrolyzing agent in the films volume during their exposure to an alkaline medium. This effect leads to film swelling and, thereby, to increases of the intensity of the destructive action of hydrolysis on the material. Chemical surface pretreatment of the nanofiller allows one to obtain a composite with an increased packing density compared to that for a composite with unmodified nanoparticles. However, the hydrolytic stability of such a film still remains somewhat inferior to that of the pristine polyimide.
\end{abstract}

Keywords: polymer-inorganic nanocomposites, polyimides, hydrolysis, carbon nanoparticles, mechanical properties, thermal stability, packing density.

Received: 27 November 2019

Revised: 29 November 2019

\section{Introduction}

Progress in the field of polymer materials science is increasingly associated with the development of new polymerinorganic nanocomposite materials [1,2]. This is due to the fact that the introduction of nanoparticles into the polymer matrix leads to the formation of the materials with improved characteristics owing to the unique properties of these nanofillers [1-4].

It is known that, depending on the geometric characteristics of the nanofiller used and the nature of its interaction with the polymer matrix, both the "physical" modification mechanism due to the reinforcement of the polymer by rigid particles with a high aspect ratio and the "chemical" modification due to the formation of additional chemical interactions in the material can take place [3-9].

Along with the aspect ratio, the extent of uniformity of nanoparticles distribution in the polymer volume is an important factor affecting the efficiency of modification the mechanical characteristics of a composite material. Most nanoparticles, due to the high surface energy, are prone to aggregation when introduced into polymer solutions and melts $[4,5]$. An effective method of solving this problem is the chemical treatment of the surface of nanoparticles before they are introduced into the polymer matrix [5]. Such a modification allows us to prevent their aggregation, to ensure the uniform distribution of nanoparticles in the polymer volume, and to improve the extent of the compatibility of nanocomposite's components.

The improvement of the characteristics of different polymer materials caused by the introduction of different nanofillers in these matrices have beenthe subject of the extensive studies over the last decade. However, most of the information obtained in these works addresses physical properties of composites under study, viz., mechanical, electric, magnetic, optical, transport, etc., whereas issues pertaining to the impact of nanosized fillers on the chemical processes in polymer matrices, are, to the best of our knowledge, poorly elucidated.

The purpose of this work is the systematic study of the impact of a number of nanoparticles, which differ both in chemical composition and in shape, upon the resistance to hydrolysis of the materials based on one of the aromatic 
polyimides (PIs) - poly-4,4'-oxydiphenylene(pyromellitimide). Films of this PI combine high hydrolytic stability with extremely high mechanical and thermal characteristics. In practice, polymers of this type are used for the manufacture of parts of devices that can be subjected to prolonged exposure to hydrolyzing agents during operation [3,10,11]. Therefore, the problem of the stability of PI-based composite materials with regard to the action of aggressive hydrolyzing media is of great practical importance. The use of nanoparticles of various types introduced into the same polymer allowed us to consider a very topical issue on the influence of specific features of the nanofiller's geometry and chemical nature both on the mechanical properties and on the hydrolytic stability of the composite material.

\section{Experimental}

Poly-4,4'-oxydiphenylene(pyromellitimide) (polyimide PMDA-ODA) was chosen as a matrix polymer to prepare the nanocomposite films:

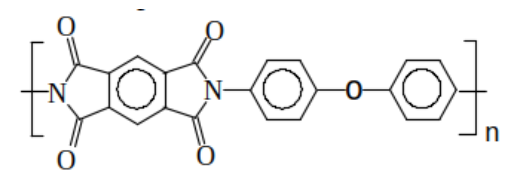

This is the most widely studied PI material, and is produced on an industrial scale (Kapton films [11] produced by DuPont and similar film materials from other producers).

Carbon nanocones/disks (CNCs), nanofibers (CNFs), multi-walled carbon nanotubes in both pristine (MWCNTs), and carboxylated (MWCNTs-COOH) states, as well as quasi-spherical cerium dioxide particles $\left(\mathrm{CeO}_{2}\right)$ were chosen as the active nano-sized fillers. CNCs, CNFs, and MWNTs are produced on an industrial scale and $\mathrm{CeO}_{2}$ particles were synthesized at the Kurnakov Institute of General and Inorganic Chemistry, Russian Academy of Sciences.

To obtain the MWCNTs-COOH the MWCNTs were subjected to surface modification before use by carboxylation in a boiling concentrated solution of nitric acid for 30 hours, followed by washing the resulting product in water to a neutral $\mathrm{pH}$ and drying [5].

The characteristics of the nanoparticles used in this work are given in Table 1.

TABLE 1. Characteristics of the nanoparticles used in the work (size, density $\rho$, producer)

\begin{tabular}{|c|c|c|c|c|c|}
\hline Nano-filler & Form & Size & $\begin{array}{c}\rho, \\
\mathrm{g} / \mathrm{cm}^{3}\end{array}$ & $\begin{array}{c}\text { Aspect } \\
\text { ratio }\end{array}$ & Producer \\
\hline \hline $\mathrm{CNF}$ & tubular & $\begin{array}{c}\text { Diameters of } 100-150 \mathrm{~nm}, \\
\text { lengths of } 6-10 \mu \mathrm{m}\end{array}$ & 2.0 & $\sim 10^{2}$ & $\begin{array}{c}\text { Showa Denko Carbon Sales } \\
\text { (USA-Japan) [13] }\end{array}$ \\
\hline $\mathrm{CNC}$ & lamellar & $\begin{array}{c}\text { Cone heights of } 0.3-0.8 \mu \mathrm{m} ; \\
\text { Diameter of cone base, } \\
\text { disc diameter of } 1-2 \mu \mathrm{m} ; \\
\text { Wall thickness of } 20-50 \mathrm{~nm}\end{array}$ & 2.0 & $\sim 10^{2}$ & n-TEC AS (Norway) [14] \\
\hline MWCNT & tubular & $\begin{array}{c}\text { External diameter of } 13 \mathrm{~nm}, \\
\text { intestine diameter of } 4 \mathrm{~nm}, \\
\text { length of }>1-20 \mu \mathrm{m}\end{array}$ & 2.1 & $\sim 10^{3}$ & $\begin{array}{c}\text { Bayer Material Science AG } \\
\text { (Germany) [15] }\end{array}$ \\
\hline $\mathrm{CeO}_{2}$ & $\begin{array}{c}\text { Quasi- } \\
\text { spherical }\end{array}$ & $\begin{array}{c}\text { Mean diameter of 3.5 nm } \\
\text { (U) }\end{array}$ & 7.65 & $\sim 1$ & $\begin{array}{c}\text { Kurnakov Institute of } \\
\text { General and Inorganic } \\
\text { Chemistry RAS (Russia) }\end{array}$ \\
\hline
\end{tabular}

The two-stage syntheticmethod was used to prepare both the nanocomposite films and control PI ones [10]. To prepare the nanocomposite films the nanoparticles of each type were introduced into the solutions of poly(amic acid) (PAA PMDA-ODA), the precursor of the PI. A solution of PAA PMDA-ODA in N,N-dimethylformamide (DMF) was provided by Sigma-Aldrich (catalog No. 575828). The nanoparticles were introduced into the polymer in the following concentrations: 3 vol.\% for CNC and CNF, and 1 vol.\% for MWCNT and MWCNT-COOH. It was shown in our previous studies $[5,12]$ being introduced into the polymer matrix in these concentrations the aforementioned nanoparticles can be uniformly distributed in the latter and thereby the increase in the Young's modulus value of the material at least by $20 \%$ can be achieved. $\mathrm{CeO}_{2}$ nanoparticles were used in the concentration of 0.55 vol. $\%$ only. Because of high density of these nanoparticles $\left(\sim 7.65 \mathrm{~g} / \mathrm{cm}^{3}\right)$ further increase in ceria concentration leads to their precipitation in the PAA solution. 
To prepare a nanocomposite solution, the calculated amount of nanoparticles was sonicated in DMF for 1 hour; the nanoparticles dispersion obtained was mixed with the required amount of PAA solution and the mixture was homogenized by a mechanical stirrer. The duration of homogenization was 24 hours.

Films of the obtained nanocomposites or of unfilled PAA were prepared by casting the flat layers of the corresponding solutions on glass substrates using a slit die with an adjustable working gap with the subsequent drying of the layers at $80{ }^{\circ} \mathrm{C}$ for 2 hours. To obtain PI and PI-based nanocomposite films, PAA and PAA-based films were cure by thermal treatment of the substrates with a heating rate of $3{ }^{\circ} \mathrm{C} / \mathrm{min}$ up to $360{ }^{\circ} \mathrm{C}$ followed by treatment at this temperature for $30 \mathrm{~min}$. The thickness of the films obtained was $30-35$ microns.

The hydrolysis of the films was performed in concentrated $(30 \%) \mathrm{NaOH}$ solution. After exposure to this solution at room temperature for different periods of time, the films were washed in distilled water to a neutral $\mathrm{pH}$ and dried.

Mechanical characteristics of the films were determined uniaxial extension using band-like samples which were $1 \mathrm{~mm}$ wide and $20 \mathrm{~mm}$ long. Experiments were carried out using AG-100kNX Plus universal mechanical tests system (Shimadzu, Japan) at the extension speed of $10 \mathrm{~mm} / \mathrm{min}$. The Young's modules $E$, yield stresses $\sigma_{y}$, tensile strengths $\sigma_{b}$, and ultimate strains $\varepsilon_{b}$ were determined by these tests. Seven samples of each type were tested with the subsequent averaging of the results obtained.

Thermomechanical analysis (TMA) for determining the glass-transition point $T_{g}$ was conducted on TMA 402 F1 Hyperion analyzer (Netzsch, Germany) with samples heated at a constant rate of $5 \mathrm{deg} \cdot \mathrm{min}^{-1}$ under a stabilized tensile stress of $0.2 \mathrm{MPa}$.

Thermogravimetric analysis (TGA) of the films was performed to determine the heat resistance indices $\tau_{5}$ and $\tau_{10}$ (the temperature values at which a polymer or a composite loses $5 \%$ and $10 \%$ of its initial weight, respectively, due to the thermal destruction processes). A DTG-60 thermal analyser (Shimadzu, Japan) was used for these tests. The samples were heated in an open crucible at a rate of $5 \mathrm{deg} \cdot \mathrm{min}^{-1}$ in a self-generated atmosphere.

The density $\rho$ values of the materials under study at a temperature of $20{ }^{\circ} \mathrm{C}$ were determined by the flotation method with small fragments of the samples in mixtures of carbon tetrachloride and toluene.

Microphotographs of the studied films surfaces were obtained using a Supra 55 VP scanning electron microscope (Carl Zeiss, Germany).

\section{Results and discussion}

The important problem to be solved while planning this investigation was to choose the criterion to be used to evaluate the intensity of the impact of hydrolyzing medium upon the materials studied.

This criterion that we have used in this work is the extent of the variation of the mechanical and thermal characteristics of the material caused by the exposure to an aqueous alkaline solution. During our preliminary investigations we have evidenced the very sensitive respond to these properties upon the hydrolytic degradation of both the pristine PI films and nanocomposite ones containing the nanofillers of different types.

First of all it was necessary to evaluate the starting level of the properties of the film materials under study. This evaluation makes it possible to compare the impact of different nanofillers upon the material's properties.

As it was repeatedly shown [1-9,12], the mechanical and thermal properties of the material are sensitive regarding to the introduction of active nanosized fillers into the polymer. Therefore, before studying the hydrolytic stability of PI-based composites, it was necessary to evaluate the degree of effectiveness of the fillers chosen as modifiers of the mechanical and thermal properties of the polymer.

The results of mechanical and thermal tests of the samples of the matrix PI film and composites based on it in the initial state are presented in the Table 2.

From the data presented it follows that not only the geometric characteristics of nanoparticles (shape, aspect ratio), but also the nature of their interaction with polymer matrix have a substantial influence on the degree of effectiveness of any filler.

CNCs were shown to be the most favourable modifier of the PI. The introduction of these nanoparticles into the material made it possible to increase the Young's modulus by $28 \%$. The introduction of two other nanofillers, namely CNF and MWCNT-COOH into PI led to a less pronounced increase in the stiffness of the material (by only $14 \%$ ). The least influence on the mechanical characteristics of PI is exerted by MWCNT and quasi-spherical nanoparticles $\mathrm{CeO}_{2}$. The introduction of $\mathrm{CeO}_{2}$ nanoparticles into the PI matrix did not produce any positive effect on the mechanical properties of the material. This situation should be expected taking into account the geometric characteristics of these nanoparticles.

Aromatic PIs are widely known as heat-resistant polymeric materials [8]. For the matrix PI used in the work, the glass transition temperature $T_{g}$ is $360-370{ }^{\circ} \mathrm{C}$, and the process of intense thermal destruction of films begins at temperature range from $500{ }^{\circ} \mathrm{C}$ and above. Composite materials containing CNC, CNF and MWCNT in the initial state are characterized by the same values of heat resistance and glass transition temperature. Only for the composite 
TABLE 2. Mechanical characteristics and glass transition temperatures of the matrix PI and nanocomposite films studied in the work

\begin{tabular}{|c|c|c|c|c|c|c|}
\hline No & Filler & $E, \mathrm{GPa}$ & $\sigma_{y}, \mathrm{MPa}$ & $\sigma_{b}, \mathrm{MPa}$ & $\varepsilon_{b}, \%$ & $T_{g},{ }^{\circ} \mathrm{C}$ \\
\hline \hline 1 & - & $2.44 \pm 0.07$ & $101 \pm 2$ & $178 \pm 3$ & $93 \pm 8$ & 370 \\
\hline 2 & $\mathrm{CNC}$ & $3.13 \pm 0.07$ & $112 \pm 2$ & $131 \pm 5$ & $29 \pm 3$ & 370 \\
\hline 3 & $\mathrm{CNF}$ & $2.78 \pm 0.07$ & $104 \pm 4$ & $116 \pm 3$ & $30 \pm 5$ & 370 \\
\hline 4 & $\mathrm{MWCNT}$ & $2.37 \pm 0.11$ & $101 \pm 2$ & $122 \pm 11$ & $33 \pm 5$ & 370 \\
\hline 5 & $\mathrm{MWCNT}-\mathrm{COOH}$ & $2.78 \pm 0.08$ & $98 \pm 1$ & $136 \pm 4$ & $62 \pm 7$ & 375 \\
\hline 6 & $\mathrm{CeO}_{2}$ & $2.49 \pm 0.05$ & $88 \pm 3$ & $103 \pm 5$ & $29 \pm 5$ & 371 \\
\hline
\end{tabular}

films containing $\mathrm{CeO}_{2}$ and MWCNT-COOH the pronounced depression of the thermal stability and some increase of the glass transition temperature were registered. The former effect is inherent to ceria-containing PMDA-ODA-based films [9].

In our previous studies $[16,17]$, we have selected the extremely severe medium to investigate the hydrolysis of PI films taking into account high hydrolytic stability of these materials. These conditions results in a rather intensive process of hydrolytic destruction of PMDA-ODA control film just in the first hours of the experiment. A marked decrease in all the mechanical characteristics of the material was observed after 1 hour of exposure in alkaline medium, and after 40 hours of hydrolysis, the film completely degrades when removed from the hydrolytic bath (Fig. 1).

The introduction of nanoparticles into PI in all cases led to a marked decrease in hydrolytic stability (Fig. 1). Films of nanocomposites filled with MWCNT completely degrade even after 3 hours of hydrolysis. The introduction of $\mathrm{CNC}, \mathrm{CeO}_{2}$, and $\mathrm{CNF}$ into the polymer matrix made it possible to obtain films slightly more resistant to hydrolysis: the complete destruction of these materials was recorded after $4-5$ hours of exposure in an alkaline solution. The film containing nanotubes subjected to a surface pretreatment, namely MWCNT-COOH is characterized by the highest resistance to hydrolytic destruction: only after $10-12$ hours of hydrolysis the film began to break into fragments, which made further research difficult.

Note that exposure in an alkaline solution leads to a successive increase in the thickness (pronounced swelling) of the samples of both PI and composites based on it (Fig. 1e). Moreover, for the composite films a successive acceleration of this process along with the increase of the hydrolysis duration was registered. The thickness of the samples was measured after washing and removing water from their surface before mechanical testing.

The impact of the hydrolysis upon the surface morphology of both PI and nanocomposite films is presented in Fig. 2.

It is known that the swelling of a polymeric material during the sorption of some aggressive medium results in a decrease of the number of inter molecular interactions in this material,resulting in a decrease in the strength of polymer products [18]. These effects were registered while analyzing the kinetics of the process of hydrolytic destruction of the films under study. The formation of micro-cracks at the films' surface registered by SEM method (Fig. 2b), undoubtedly facilitates the propagation of the aggressive medium - alkaline solution into the polymer volume. The mechanical stresses caused by the incorporation of this solution into the cracks of the in homogeneously swollen material provoke the further destruction of the films [19]. The nanocomposite films, along with the aforementioned effect's tendency to accelerate the decomposition, the additional cracking at the boundaries "polymer-nanoparticles" with the formation of pores at these regions (the inferent SEM picture of the surface of nanocomposite containing CNCs exposed to hydrolysis during $3 \mathrm{~h}$ is presented in Fig. 2d). The micro- and nano-sized inhomogeneities formed at the phase boundaries during the nanocomposite films production because of the imperfect compatibility of the polymer and nanoparticles can give rise to these pores.

To elucidate the extent of the compatibility of the nanoparticles with the PI matrix, insured during the formation of composite films, the expected density values of the nanocomposite films under study were calculated by using the additive scheme of composite formation [7]: 

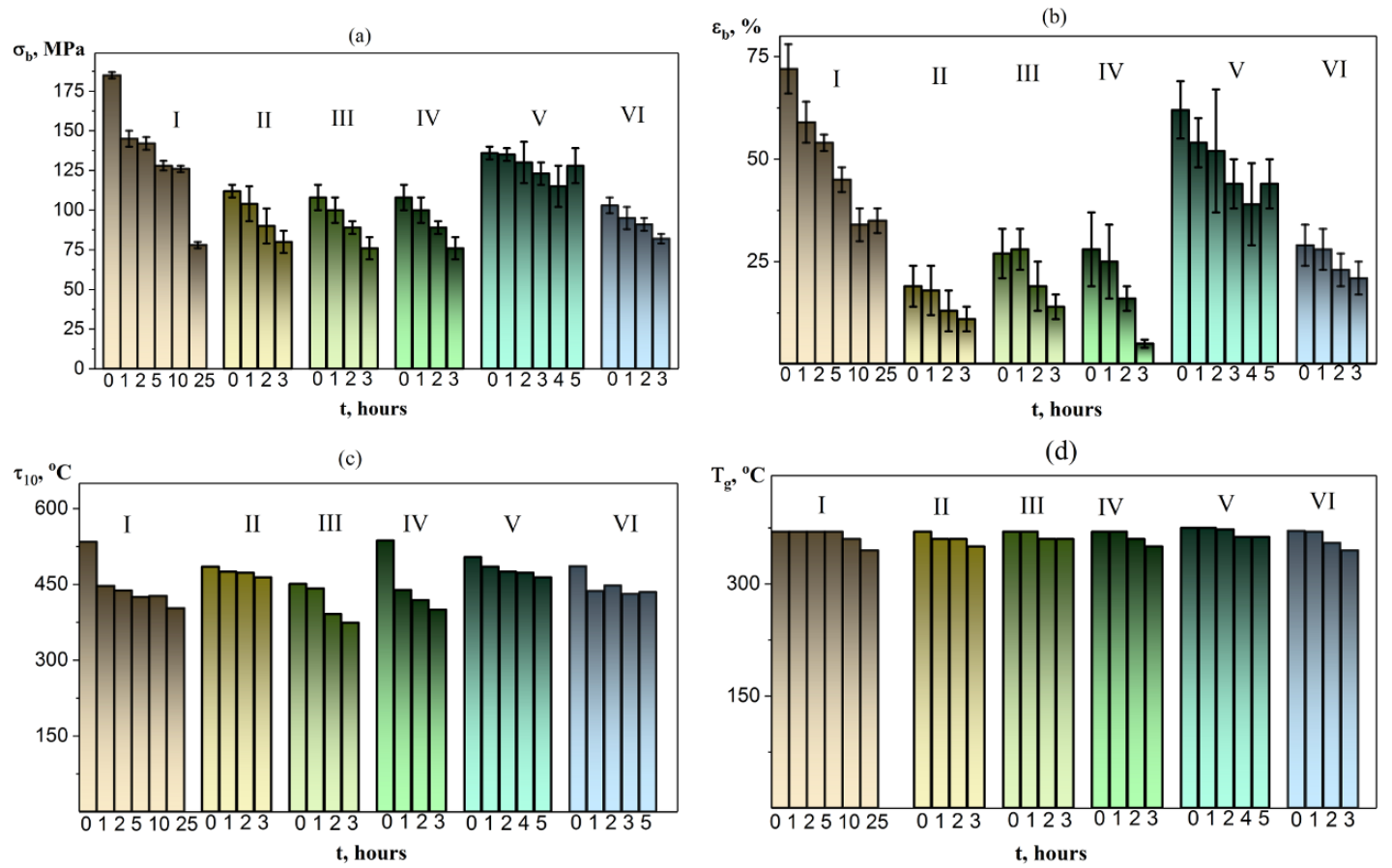

(d)
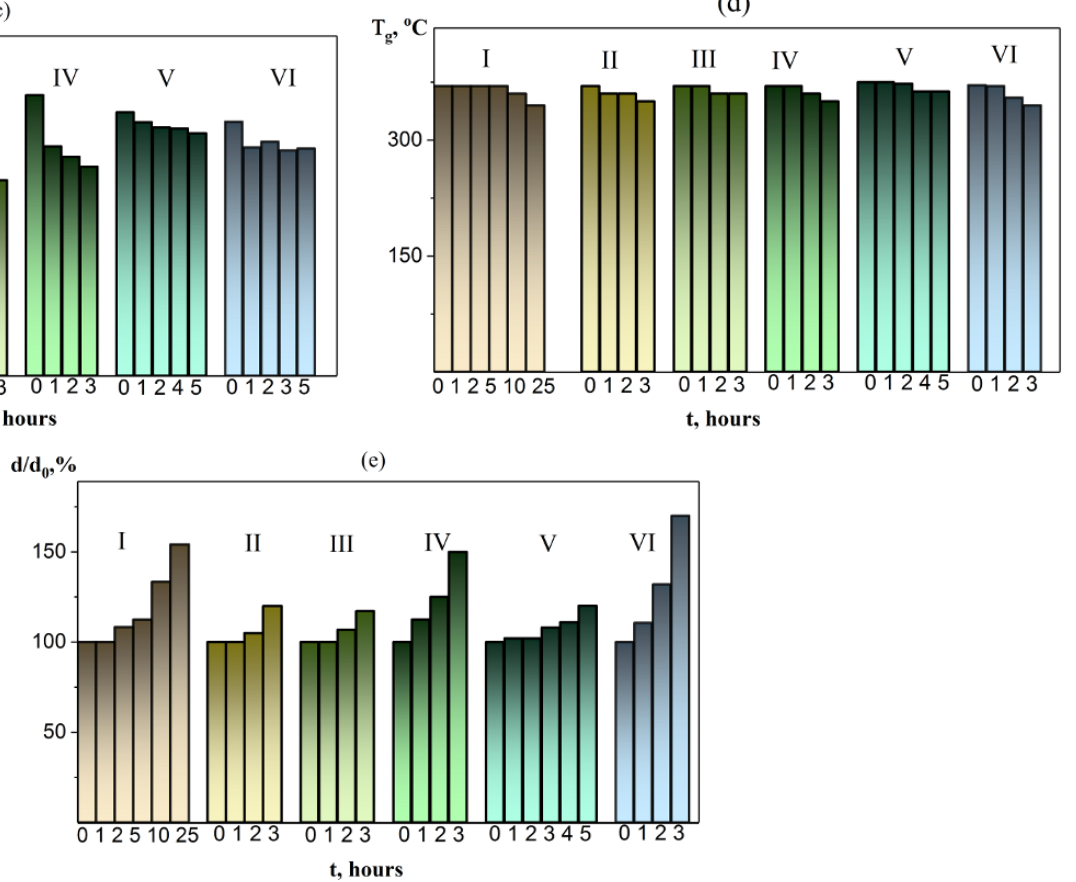

FIG. 1. (a) Tensile strength $\sigma_{b}$, (b) ultimate strain $\varepsilon_{b}$, (c) glass transition temperature $T_{g}$, (d) heat resistance index $\tau_{10}$, (e) thickness $d / d_{0}$, of the films tested vs. the hydrolysis duration: (I) unfilled PI, (II) PI-CNC, (III) PI-CNF, (IV) PI-MWCNT, (V) PI-MWCNT-COOH, and (VI) PI-CeO ${ }_{2}$; $d_{0}$ stands for the initial thickness if the film tested

$$
\rho_{\text {calc }}=\rho_{P I} \times C_{P I}+\rho_{\text {nano }} \times C_{\text {nano }},
$$

where $\rho_{\text {calc }}$ is the density of an "ideal" nanocomposite containing no pores; $\rho_{P I}$ and $\rho_{\text {nano }}$ denote the densities of the polymer and the nanoparticles introduced into it; whereas $C_{P I}$ and $C_{n a n o}$ denote their volume fractions in the nanocomposite, respectively.

As it was noted in [7], this calculation does not provide precise values of density of the nanocomposite materials under consideration because of the rather poor accuracy of the information concerning the nanoparticles' density values (producer's data). However, these results can give the reliable estimation of the tendency to the variation of free volume caused by the introduction of the nanoparticles into polymer.

The experimental density values of all studied nanocomposites were found to be inferior to calculated values (Table 4). This result testifies to the presence of a certain excess free volume in the composite films. Its value is sufficiently small: the ratio of the experimental density of the sample to the calculated one in all cases wasn't less than 0.994. The maximum value of this ratio corresponds to a composite containing carboxylated nanotubes $\left(\rho_{\text {exp }} / \rho_{\text {calc }}=\right.$ 0.999, Table 4$)$. The composites containing $\mathrm{CeO}_{2}\left(\rho_{\text {exp }} / \rho_{\text {calc }}=0.998\right), \mathrm{CNC}$ and $\mathrm{CNF}\left(\rho_{\text {exp }} / \rho_{\text {calc }}=0.996\right)$ are characterized by slightly less dense packing. The highest value of the excess free volume was observed for the PIMWCNT composite $\left(\rho_{\text {exp }} / \rho_{\text {calc }}=0.994\right)$. 

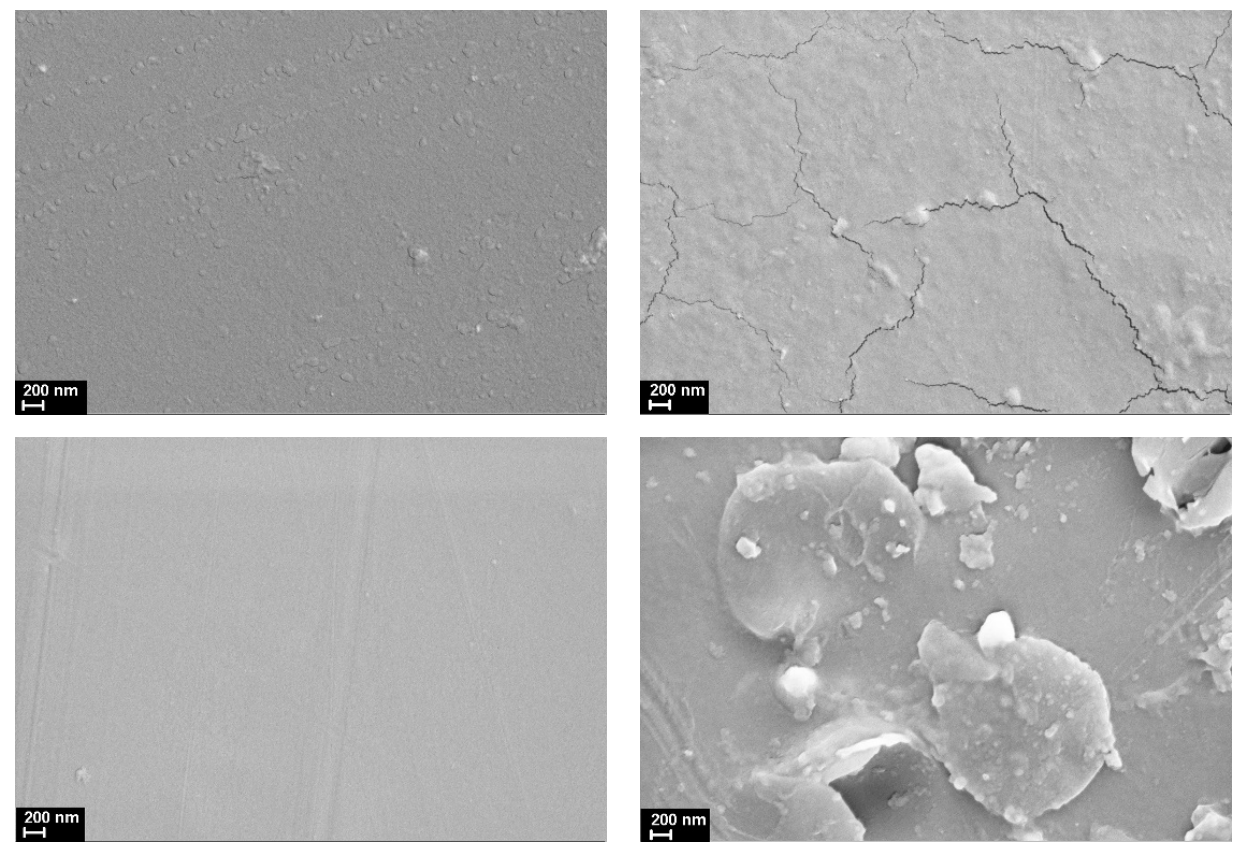

FIG. 2. SEM images of surfaces of the films: (a) PI in the initial state, and (b) after 25 hours of hydrolysis; (c) PI-CNC composite in the initial state, and (d) after 3 hours of hydrolysis

TABLE 3. Thermal stability of the matrix and nanocomposite films investigated in the work

\begin{tabular}{|c|c|c|c|}
\hline No & Filler & $\tau_{5},{ }^{\circ} \mathrm{C}$ & $\tau_{10},{ }^{\circ} \mathrm{C}$ \\
\hline \hline 1 & - & 505 & 534 \\
\hline 2 & $\mathrm{CNC}$ & 506 & 529 \\
\hline 3 & $\mathrm{CNF}$ & 497 & 524 \\
\hline 4 & $\mathrm{MWCNT}$ & 506 & 537 \\
\hline 5 & $\mathrm{MWCNT}-\mathrm{COOH}^{2}$ & 470 & 504 \\
\hline 6 & $\mathrm{CeO}_{2}$ & 370 & 425 \\
\hline
\end{tabular}

TABLE 4. Experimental and calculated density values of the nanocomposite films studied in the work

\begin{tabular}{|c|c|c|c|}
\hline Filler & $\rho_{\text {exp }}, \mathrm{g} / \mathrm{cm}^{3}$ & $\rho_{\text {calc }}, \mathrm{g} / \mathrm{cm}^{3}$ & $\rho_{\text {exp }} / \rho_{\text {calc }}$ \\
\hline \hline $\mathrm{CNC}$ & 1.423 & 1.428 & 0.996 \\
\hline $\mathrm{CNF}$ & 1.423 & 1.428 & 0.996 \\
\hline $\mathrm{MWCNT}$ & 1.408 & 1.417 & 0.994 \\
\hline $\mathrm{MWCNT}-\mathrm{COOH}$ & 1.416 & 1.417 & 0.999 \\
\hline $\mathrm{CeO}_{2}$ & 1.441 & 1.444 & 0.998 \\
\hline
\end{tabular}


As it was shown in previous studies $[16,17]$ and confirmed by the described work, a clear correlation is observed between the excess free volume determined in the densitometric experiment and extent of the hydrolytic stability of composites.

It would be interesting to trace such correlation by analysing the results obtained during the mechanical tests of the composite films. According to previously obtained data [7], the degree of intensity of the impact of nanoparticles introduced into the PI matrix upon the mechanical characteristics of the material (the difference between the properties of the nanocomposite and the initial PI) decreases along with the increase of the excess free volume formed during the formation of the nanocomposite material. Indeed, the main reason of the formation of excess free volume in a nanocomposite material is the incomplete compatibility of the nanoparticles with the polymer matrix. This lack of compatibility leads to a decrease in the local density of the material in the phase boundaries. The described effect naturally produces a negative influence on the mechanical characteristics of the material. However, the results of our tests are not entirely consistent with the above consideration. Indeed, it follows from the comparison of the calculated and experimental density values that a very dense and defect-free packing is realized in a nanocomposite film filled with cerium oxide (the ratio of the experimental density to the calculated one is 0.998 ). But the introduction of just these nanoparticles into the PI matrix led to a minimal increase in film stiffness (Table 4).

In fact, this apparent contradiction is caused by the well-known fact: the intensity of the variation of the mechanical properties of a matrix polymer caused by the introduction of some nanofilleris determined not only by the intensity of interaction of the composite's components, but also by ability of the nanoparticles used to create a rigid carcass inside the polymer volume. This frame can bear the mechanical load applied to the material and distribute it in the volume of the sample [1,2]. The effectiveness of the impact of this framework on the properties of the material increases along with the increase in the aspect ratio of the nanoparticles introduced into the polymer [1,2, 20]. For quasispherical cerium oxide nanoparticles, the value of the aspect ratio is close to 1 , while for other nanofillers used in the work, it is $2-3$ orders of magnitude higher. Given this circumstance, the result of mechanical tests of composites with cerium oxide should be treated as quite regular.

\section{Conclusions}

Based on the data presented, the following main conclusion can be drawn: the introduction of the nanoparticles of different types into the poly(pyromellitimide) matrix, regardless of their shapes and chemical nature, along with some positive effects, can provoke a decrease in the hydrolytic stability of the material. This effect is caused by the increase in the excess free volume in the process of the nanocomposite formation. The excessive free volume facilitates the diffusion of the hydrolyzing agent into the volume of the film during its aging in an alkaline medium (film swelling) that causes an increase in the extent of destructive action of the hydrolytic attack on the material. It is possible to increase the packing density of the composite due to the surface functionalization of the nanoparticles before their introduction into the polymermatrix. This enhancement of the material's density will insure the decrease in its permeability to hydrolyzing solution. In our work, this effect was realized by the chemical pretreatment (carboxylation) of MWCNT: while these nanoparticles were used as nanofiller the material was formed with the increased packing density as compared to that of a composite filled with the untreated MWCNT. However, even in this case, the hydrolytic stability of the composite film still remains somewhat lower than that of the initial PI.

\section{Acknowledgements}

The authors are grateful to M. Ya. Goikhman and I. V. Abalov (Institute of Macromolecular Compounds, Russian Academy of Sciences), who provided MWCNTs for this work.

This work was supported by the Russian Science Foundation (project No. 18-13-00305).

\section{References}

[1] Gogotsi Y. Nanomaterials handbook. Tailor and Francis, Boca Raton, London, New York, 2006, 779 p.

[2] Zaman I., Manshoor B., Khalid A., Araby S. From clay to graphene for polymer nanocomposites - a survey. J. Polym. Res., 2014, 5 (21), P. 1-11.

[3] Kausar A. Progression from polyimide to polyimide composite in proton-exchange membrane fuel cell: a review. Polymer-Plastics Technology and Engineering, 2017, 56 (13), P. 1375-1390.

[4] Eletskii A.V., Knizhnik A.A., Potapkin B.V., Kenny J.M. Electrical characteristics of carbon nanotube-doped composites. Physics-Uspekhi, 2015, 58 (3), P. 209-251.

[5] Gofman I.V., Abalov I.V., et al. Comparative Evaluation of Different Methods of Carboxylation of Carbon Nanotubes as a Modifier of Mechanical Properties of Heat-Resistant Polyimide Based Nanocomposites. Fibre Chemistry, 2015 , 47 (4), P. $236-243$.

[6] Shang Z., Lu C., Lü X., Gao L. Studies on syntheses and properties of novel $\mathrm{CeO}_{2} /$ polyimide nanocomposite films from Ce(Phen) 3 complex. Polymer, 2007, 48 (14), P. 4041-4046. 
[7] Gofman I.V., Ivankova E.M., et al. Effect of nanoparticles of various types as fillers on mechanical properties of block samples of a heatresistant polyimide material: A comparative analysis. Polymer Science Series A, 2016, 58 (1), P. 87-94.

[8] Pandey R.P., Amit K.T., Shahi V.K. Sulfonated polyimide/acid-functionalized graphene oxide composite polymer electrolyte membranes with improved proton conductivity and water-retention properties. ACS applied materials \& interfaces, 2014,6 (19), P. 16993-17002.

[9] Gofman I., Nikolaeva A., et al. Unexpected selective enhancement of the thermal stability of aromatic polyimide materials by cerium dioxide nanoparticles. Polymers for advanced technologies, 2019, 30 (6), P. 1518-1524.

[10] Bessonov M.I., Koton M.M., Kudryavtsev V.V., Laius L.A. Polyimides - Thermally Stable Polymers. New York: Plenum Publishing Corp., 1987. $374 \mathrm{p}$.

[11] URL: http://www.dupont.com/products-and-services/membranes-films/polyimide-films/brands/ kapton-polyimide-film.html.

[12] Gofman I.V., Abalov I.V., Tiranov V.G., Yudin V.E. Effect of carbon nanoparticles of different shapes on mechanical properties of aromatic polyimide-based composite films. Polymer Science Series A, 2013, 55 (5), P. 313-319.

[13] URL: https://www.sdk.co.jp/english/products/126/132/2094.html.

[14] URL: https://www.strem.com/uploads/resources/documents/nanocones.pdf.

[15] URL: https://ru.scribd.com/document/59272724/Bay-Tubes.

[16] Bykova E.N., Gofman I.V., Vlasova E.N. Hydrolytic stability of films of aromatic polyimides and composites on their basis, filled with carbon nanocones. Russian Journal of Applied Chemistry, 2018, 91 (9), P. 1460-1470.

[17] Bykova E.N., Gofman I.V. Effect of nanosized carbon fillers on the hydrolytic stability of films of a heat-resistant aromatic polyimide. Russian Journal of Applied Chemistry, 2017, 90 (1), P. 70-76.

[18] Rudakova T.E., Zaikov G.E. Degradation of polymers by mechanical forces and chemically active media. Polymer degradation and stability, 1988, 21 (1), P. 73-90.

[19] Malkin A.I. Regularities and mechanisms of the Rehbinders effect. Colloid Journal, 2012, 2 (74), P. $223-238$.

[20] Garboczi E.J., Snyder K.A., Douglas J.F., Thorpe M.F. Geometrical percolation threshold of overlapping ellipsoids. Phys. Review E, 1995, 1 (52), P. 819-828. 